\title{
DIVERSIDADE POPULACIONAL DE ARTRÓPODES NA CULTURA DA ABOBRINHA UTILIZANDO ARMADILHAS DO TIPO MOERICK E PITFALL
}

Daniel Della Libera ${ }^{1}$, Siumar Pedro Tironi ${ }^{2}$, André Luiz Radunz ${ }^{3}$, Marco Aurélio Tramontin $^{4}$

1 Engenheiro Agrônomo, Graduado em Agronomia, Universidade Federal da Fronteira Sul

2 Professor Doutor do Departamento de Agronomia da Universidade Federal da Fronteira Sul

3 Professor Doutor do Departamento de Agronomia da Universidade Federal da Fronteira Sul

4 Professor Doutor do Departamento de Agronomia da Universidade Federal da Fronteira Sul (marco.silva@uffs.edu.br) Chapecó-Brasil

Recebido em: 15/04/2017 - Aprovado em: 22/07/2017 - Publicado em: 31/07/2017 DOI: 10.18677/Agrarian_Academy_2017a16

\begin{abstract}
RESUMO
A abobrinha italiana é cultivada em todo o território nacional e bastante consumida devido as suas características nutricionais e a sua praticidade e facilidade de preparo. Os insetos apresentam grande importância na cultura da abobrinha italiana, pois estas dependem de insetos para realizar a polinização, além disso, insetos podem causar danos às plantas e aos frutos. Assim, objetivou-se realizar uma avaliação da entomofauna presente na cultura da abobrinha durante seu ciclo. Foram implantados quatro canteiros de abobrinha italiana e durante seu ciclo, foram realizadas três capturas de artrópodes com armadilhas Pitfall e três coletas com armadilhas Moericke. As coletas foram realizadas quando as plantas estavam com 18,46 e 65 dias de campo. Em cada coleta foram utilizadas duas armadilhas, de cada tipo totalizando seis armadilhas do tipo Pitfall e seis armadilhas do tipo Moericke. Após a coleta, os insetos foram classificados a nível de ordem. No estudo realizado, a ordem Hymenoptera apresentou a maior quantidade de insetos capturados, e totalizou 631 indivíduos. A armadilha do tipo Moericke capturou maior diversidade de insetos no início do desenvolvimento da cultura apresentando índice de Shannon de 1,61. A armadilha do tipo Pitfall apresentou maior diversidade de insetos quando a cultura estava em plena floração, e o índice de Shannon foi de 1,30. As armadilhas utilizadas no experimento foram eficientes na captura de artrópodes das ordens Coleoptera, Collembola, Diptera, Hemiptera e Hymenoptera A ordem que teve maior número de indivíduos capturados foi a ordem Hymenoptera, representando $48,91 \%$ dos artrópodes amostrados.
\end{abstract}

PALAVRAS-CHAVE: Collembola, Cucurbitaceae, Hymenoptera. 


\title{
POPULATIONAL DIVERSITY OF ARTHROPODS IN ZUCCHINI CROP USING MOERICK AND PITFALL TRAPS
}

\begin{abstract}
The italian zucchini is cultivated throughout the country and widely consumed because of their nutritional characteristics and their practicality and ease of preparation. The insects have great importance in italian zucchini crop as these rely on insect pollination to perform, moreover, insects may cause damage to plants and fruits. Thus, we aimed to carry out an assessment of this entomofauna in the crop of italian zucchini during their cycle. To conduct this study, four squares sites were deployed and during their cycle, there were three collections of arthropods with Pitfall traps and three collections with Moericke traps. Samples were collected when the plants were 18, 46 and 65 field days. In each survey was used two traps of each type, being six traps type Pitfall and six traps type Moericke. After collection, the insects were classified at the order level. In the study, the Hymenoptera order presented the highest amount of captured insects, totaling 631 individuals. The trap type Moericke captured greater insect diversity early in the development of crop with a Shannon index of 1.61. The Pitfall trap showed greater insect diversity when the crop was in full bloom, the Shannon index was 1.30. The traps used in the experiment were efficient in arthropods capture of Coleoptera, Collembola, Diptera, Hemiptera and Hymenoptera. The order that had the largest number of individuals captured was the order Hymenoptera, representing $48,91 \%$ of the arthropods sampled.
\end{abstract}

KEYWORDS: Collembola, Cucurbitaceae, Hymenoptera.

\section{INTRODUÇÃO}

O Brasil produz aproximadamente 158 mil toneladas de abobrinha italiana (Cucurbita pepo L.), predominantemente em propriedades de até 50 hectares. No estado de Santa Catarina esta espécie produzida em 948 propriedades (IBGE, 2009). Este cenário, associado às características de cultivo como o ciclo curto, rápido retorno econômico e as possibilidades de agroindustrialização, demonstram que a cultura da abobrinha possui potencial para ser inserida na diversificação da matriz produtiva das unidades familiares, enaltecendo sua importância socioeconômica nacional.

Durante o ciclo de cultivo da abobrinha, problemas fitossanitários podem ocorrer, entre os quais, se destacam os insetos-praga. Entre estes os pulgões, as brocas, as vaquinhas e as lagartas destacam-se como os principais, os quais se alimentam de suas partes ou dos fotoassimilados. Neste sentido, estratégias de manejo de insetos-praga que acometem a cultura da abobrinha são necessárias, sendo esta em geral adotada pelo uso dos inseticidas químicos, os quais podem apresentar aspectos negativos ao ambiente e à saúde dos consumidores e agricultores (MASCARENHAS et al., 2007).

Assim, para implantar sistemas que minimizam a utilização de agrotóxicos, como exemplo, o Manejo Integrado de Pragas (MIP), torna-se fundamental conhecer a entomofauna do local de cultivo, a fim de identificar os insetos-praga, mas também a diversidade de inimigos naturais e polinizadores (MICHEREFF FILHO et al., 2012).

Neste contexto, as armadilhas para o monitoramento de artrópodes em AGRARIAN ACADEMY, Centro Científico Conhecer - Goiânia, v.4, n.7; p. 177 2017 
agroecossistemas tem sido amplamente empregadas. Entre estas pode se destacar a tipo Moericke, utilizada, entre outros, em levantamentos realizados na cultura da soja (LARA et al., 2009), na cultura do cafeeiro (LARA et al., 2011; TANGO et al., 2014), coco (COMERIO et al., 2013) e citros (LARA et al., 2015). E também as armadilhas do tipo Pitfall, comumente utilizadas para estudos com a meso e a macrofauna do solo as quais têm baixo custo, são fáceis de instalar e amplamente empregadas em trabalhos de inventário de biodiversidade (MOREIRA et al., 2010; SILVA \& AMARAL, 2013; LARA et al., 2015).

Apesar da conhecida eficiência da utilização individualizada das armadilhas tipo Moericke e Pitfall, tem sido comum a indicação do uso combinado destas em especial pela obtenção de capturas de insetos mais diversificadas. Pelo exposto, objetivou-se avaliar a população da entomofauna da cultura da abobrinha italiana (Cucurita pepo L.) utilizando armadilhas do tipo Pitfall e Moericke.

\section{MATERIAL E MÉTODOS}

\section{Local de amostragem}

As amostragens dos indivíduos foram realizadas na área experimental da Universidade Federal da Fronteira Sul (UFFS), Campus Chapecó, Santa Catarina, latitude -27ำ11'89", e longitude -52ํㅜㅇ'56".

\section{Obtenção e condução da cultura}

As mudas da abobrinha (Cucurbita pepo L.) foram obtidas comercialmente no município de Chapecó, Santa Catarina, e transplantadas em quatro canteiros localizados na área experimental da UFFS. Cada canteiro apresentava 14 metros de comprimento e 1,20 metros de largura. Um espaçamento de 0,30 metros foi mantido entre os canteiros. As mudas de abobrinha estavam espaçadas entre si por 1,0 metro. Irrigações foram realizadas de forma manual até os 14 dias da cultura no campo.

\section{Amostragem de indivíduos}

Cada coleta foi composta por oito armadilhas Moericke e oito armadilhas Pitfall, sendo que posteriormente foram sorteadas duas armadilhas aéreas Moericke e duas armadilhas de solo Pitfall de cada coleta para triagem, e assim totalizando 12 armadilhas analisadas em três coletas. As armadilhas de solo foram confeccionadas utilizando garrafas Pet com $15 \mathrm{~cm}$ de altura e 10,5 cm de diâmetro, e continham em seu interior uma solução com água, detergente (5\%) e água sanitária (3\%). As armadilhas aéreas foram confeccionadas utilizando pratos plásticos de sobremesa com $13 \mathrm{~cm}$ de diâmetro na coloração amarela, juntamente com palitos de madeira, unidos com cola quente. Para a instalação das armadilhas aéreas (Moericke) nos canteiros, fez- se o uso de estacas de bambu fixadas a 1 metro de altura em relação ao solo, nas quais as armadilhas foram fixadas com auxílio de fita adesiva. Em seu interior foi feito uso de uma solução com água, detergente (5\%) e água sanitária (3\%). As armadilhas permaneceram dois dias nos canteiros, sendo a primeira coleta realizada entre os dias 14 a 16 de outubro, a segunda de 11 a 13 de novembro e a última de 30 de novembro a dois de dezembro de 2015. As coletas corresponderam ao $18^{\circ}, 46^{\circ}$ e $65^{\circ}$ dias após o transplantio da abobrinha.

\section{Identificação dos indivíduos}

O material coletado foi etiquetado e armazenado em álcool 70\% e colocado AGRARIAN ACADEMY, Centro Científico Conhecer - Goiânia, v.4, n.7; p.178 2017 
em sacolas plásticas de dois litros e acondicionados em caixas de poliestireno e levados ao laboratório de Botânica, Ecologia e Entomologia da UFFS, Campus Chapecó, Santa Catarina. Posteriormente, os invertebrados foram retirados das embalagens em que se encontravam armazenados e, com o auxílio de pinça e pincel, foram identificados com auxílio de microscópio estereoscópico.

\section{Análise dos dados}

Todas as análises estatísticas basearam-se em dados quantitativos, considerando os indivíduos coletados nas armadilhas aleatoriamente selecionadas para identificação ao táxon de Ordem. O índice de diversidade (Shannon's index) foi calculado usando o Programa R versão 3.2.3 (2016) The R Foundation for Statistical Computing. Os dados meteorológicos foram obtidos na Epagri localizada no município de Chapecó, Santa Catarina. O índice de Shannon foi calculado e então os dados analisados.

\section{RESULTADOS E DISCUSSÃO}

\section{Ordens coletadas na cultura da abobrinha}

Durante o período do experimento, foram coletados 1290 invertebrados, sendo os artrópodes identificados como pertencentes a 11 ordens distintas com a respectiva quantidade de indivíduos coletados em ambas armadilhas: Araneae (23), Blattodea (1), Coleoptera (118), Collembola (257), Dermaptera (1), Diptera (155), Hemiptera (44), Hymenoptera (631), Lepidoptera (7), Orthoptera (22) e Thysanoptera (31) (Tabela 1). As Ordens encontradas nessa avaliação populacional, pertencem as principais em importância agrícola, sendo encontradas em pesquisas sobre entomofauna realizadas em diversas culturas (MOREIRA et al., 2010).

TABELA 1 - Quantidade (№) e frequência relativa (FR\%) dos artrópodes coletadas nas armadilhas Pitfall e Moericke, em três datas de coletas na cultura da abobrinha italiana, Chapecó, 2015.

\begin{tabular}{lllllll}
\hline \multirow{2}{*}{ Ordem } & \multicolumn{2}{l}{ Armadilha tipo Pitfall } & \multicolumn{2}{l}{ Armadilha tipo Moericke } & \multicolumn{2}{l}{$\begin{array}{l}\text { Total armadilhas } \\
\text { Pitfall e Moericke }\end{array}$} \\
\cline { 2 - 7 } & № & FR\% & № & FR\% & No & FR\% \\
\hline Araneae & 20 & 2,07 & 3 & 0,92 & 23 & 1,78 \\
Blattodea & 1 & 0,1 & 0 & 0 & 1 & 0,08 \\
Coleoptera & 65 & 6,74 & 53 & 16,26 & 118 & 9,15 \\
Collembola & 257 & 26,66 & 0 & 0 & 257 & 19,92 \\
Dermaptera & 1 & 0,1 & 0 & 0 & 1 & 0,08 \\
Diptera & 24 & 2,49 & 131 & 40,18 & 155 & 12,02 \\
Hemiptera & 26 & 2,7 & 18 & 5,52 & 44 & 3,41 \\
Hymenoptera & 543 & 56,33 & 88 & 26,99 & 631 & 48,91 \\
Lepidoptera & 0 & 0 & 7 & 2,15 & 7 & 0,54 \\
Orthoptera & 22 & 2,28 & 0 & 0 & 22 & 1,71 \\
Thysanoptera & 5 & 0,52 & 26 & 7,98 & 31 & 2,4 \\
\hline Total & 964 & 100 & 326 & 100 & 1290 & 100 \\
\hline
\end{tabular}

AGRARIAN ACADEMY, Centro Científico Conhecer - Goiânia, v.4, n.7; p.179 2017 
A predominância na ocorrência de indivíduos pertencentes às ordens Hymenoptera, Collembola, Diptera e Coleoptera, capturados na cultura da abobrinha, provavelmente esteja associada a maior quantidade de espécies que compõe essas ordens, bem como, devido ao tipo de armadilha utilizada (GALLO et al., 2002). A ordem Hymenoptera é uma das mais importantes do ponto de vista agrícola, especialmente por conter a maioria dos polinizadores de plantas cultivadas, mas também as espécies consideradas pragas, como as formigas e a vespa da madeira. Além desses, apresenta também diversos inimigos naturais (GALLO et al., 2002). Já, a baixa ocorrência das ordens Dermaptera e Blattodea, as quais apresentaram apenas um indivíduo capturado nas armadilhas, pode ser explicado pela sua preferência por locais fechados e escuros (GARCIA, 2008).

A diversidade da população de indivíduos pode ser definida como 0 número de espécies que são encontradas em uma amostra retirada de uma área em determinado período. Neste sentido, a diversidade é expressa na Tabela 2, através do índice de Shannon.

TABELA 2 - Dias de coletas e índice de diversidade de Shannon para os dois tipos de armadilhas.

\begin{tabular}{lll}
\hline & \multicolumn{2}{c}{ Indice de Shannon } \\
\cline { 2 - 3 } Coleta & Arm. Moericke & Arm. Pitfall \\
\hline Primeira (18 dias) & 1,61 & 1,13 \\
Segunda (46 dias) & 1,47 & 1,30 \\
Terceira (65 dias) & 1,09 & 1,21 \\
\hline Diversidade Total & 1,50 & 1,25
\end{tabular}

A armadilha Moericke apresentou um índice de Shannon maior que as armadilhas do tipo Pitfall. Isso ocorreu, uma vez que, com menor número de indivíduos capturados, conseguiu amostrar diversas ordens de insetos. As armadilhas Pitfall capturaram mais indivíduos, porém não significou que estes representassem maior número de ordens, ou que, o número mais alto de artrópodes capturados representassem proporcionalmente mais ordens se comparado às armadilhas do tipo Moericke.

Ao comparar as coletas dentro do mesmo tipo de armadilha (Moericke e Pitfall), as armadilhas do tipo Moericke instaladas na primeira coleta apresentaram a maior diversidade. Isso pode ter ocorrido pelo fato de alguns tipos de insetos se beneficiarem com a cultura da abobrinha e, passaram a ter seu crescimento populacional favorecido em detrimento dos insetos das demais ordens. $E$ isso pode ter ocasionado a diminuição gradual da diversidade nas armadilhas do tipo Moericke.

Nas armadilhas de solo, Pitfall's, a diversidade de insetos foi maior na segunda coleta, e isso indica que o desenvolvimento da cultura da abobrinha atraiu uma diversidade maior de insetos quando comparado com a cultura quando recém implantada. 


\section{Fauna de Hymenoptera}

A ocorrência da ordem Hymenoptera pode ser atribuída à presença de grande quantidade de insetos polinizadores, insetos estes que são fundamentais para que a abobrinha venha a produzir frutos de qualidade. A ordem Hymenoptera foi a que apresentou maior número de indivíduos capturados na presente pesquisa, tendo apresentado elevado número de indivíduos da família formicidae nas armadilhas do tipo Pitfall. O número de indivíduos da família formicidae capturados representou cerca de $40 \%$ do total de insetos capturados nesse experimento e, cerca de $54 \%$ do total de insetos capturados nas armadilhas do tipo Pitfall, somando 526 indivíduos. Esta ocorrência pode ser atribuída a liberação de feromônios, por parte das formigas, sempre que estas estão em uma situação de perigo, o que proporcionou maior atração dos indivíduos para a armadilha (VILELA, 1994).

A captura de formigas no presente experimento é um indicativo positivo, uma vez que, estes insetos possuem papel importante na ciclagem de nutrientes, decomposição de material orgânico, dispersão de sementes e predação de outros artrópodes. Nas armadilhas do tipo Moericke, a captura de indivíduos da Ordem Hymenoptera foi influenciada pela data de coleta, tendo sido maior na segunda coleta, 5,7 vezes superior a primeira data, fato que provavelmente esteja associado a fase de floração das plantas de abobrinha, a qual coincidiu com a data de avaliação das armadilhas. Contudo, já na terceira coleta, foram capturados apenas sete indivíduos, o que representa pouco mais de $10 \%$ da segunda coleta.

\section{Fauna de Collembola}

As armadilhas do tipo Pitfall capturaram grande quantidade de artrópodes da ordem Collembola, no total, 257 indivíduos, tendo sido capturados 77, 131 e 49, na primeira, segunda e terceira coleta, respectivamente. A grande representação no número de indivíduos capturados pode ser explicada pelo fato desses artrópodes serem um dos mais abundantes da fauna do solo. Ordem esta que contempla importantes representantes para o equilíbrio do solo, pois podem se alimentar de fungos e ciclar nutrientes, visto que consome bactérias e decompõe restos vegetais e animais. Os colêmbolos são considerados animais da base da cadeia trófica, uma vez que servem de alimentos para diversos insetos e aracnídeos (BELLINI \& ZEPPELINI, 2009).

\section{Fauna de Diptera e Coleoptera}

Ao total, foram coletados 155 dípteros, destes, 24 foram capturados nas armadilhas do tipo Pitfall e 131 nas armadilhas do tipo Moericke. A ordem Diptera, para agroecossistemas agrícolas, apresenta importância significativa, tendo em vista que possui entre seu representantes, pragas importantes para a maioria das culturas, como exemplo, a mosca-das-frutas, mosca minadora e mosca-do-sorgo. Bem como, encontram-se os insetos que atuam como polinizadores.

No que tange a ordem Coleoptera, foram capturados 118 insetos, sendo 65 nas armadilhas do tipo Pitfall e 53 nas armadilhas do tipo Moericke. A ordem Coleoptera é constituída por besouros, estes insetos podem causar grandes prejuízos às culturas (vaquinhas, brocas) mas, também, apresentam inimigos naturais muito importantes, como as joaninhas (GARCIA, 2008). 


\section{Flutuações populacionais}

Ao analisar os dados das coletas, observou-se que houve uma flutuação na quantidade de indivíduos capturados. A variação ocorreu principalmente no número total de indivíduos e o número de indivíduos por ordem. Assume-se que diversos fatores possam ser os responsáveis por esta variação, entre os quais estão o ciclo da cultura (fases fenológicas distintas ao longo das avaliações) e também as condições climáticas presentes nos momentos da coleta dos insetos.

Neste sentido, destaca-se ainda que os principais fatores que interferem nas espécies de insetos encontrados em um determinado local são a radiação solar, temperatura do ar e do solo, umidade do solo e relativa do ar, precipitação pluvial e a velocidade do vento. Assim, a ênfase da discussão será feita relacionando a flutuação populacional com os dados de temperatura do ar e de precipitação pluvial.

Durante a condução do experimento, meses de setembro a dezembro, a variação de temperatura máxima e mínima do ar foi de $3 \div \mathrm{C}$ e a precipitação pluvial foi de $274,8,209,2,335,2$ e $376,4 \mathrm{~mm}$, respectivamente nos meses de setembro, outubro, novembro e dezembro. Neste contexto, Gallo et al.(2002) destacam que a precipitação pluvial afeta principalmente insetos de solo, pois estes são influenciados pela disponibilidade de água no solo. Contudo, ao analisar a figura 1, não foi possível estabelecer uma correlação entre os fatores indivíduos capturados e precipitação.

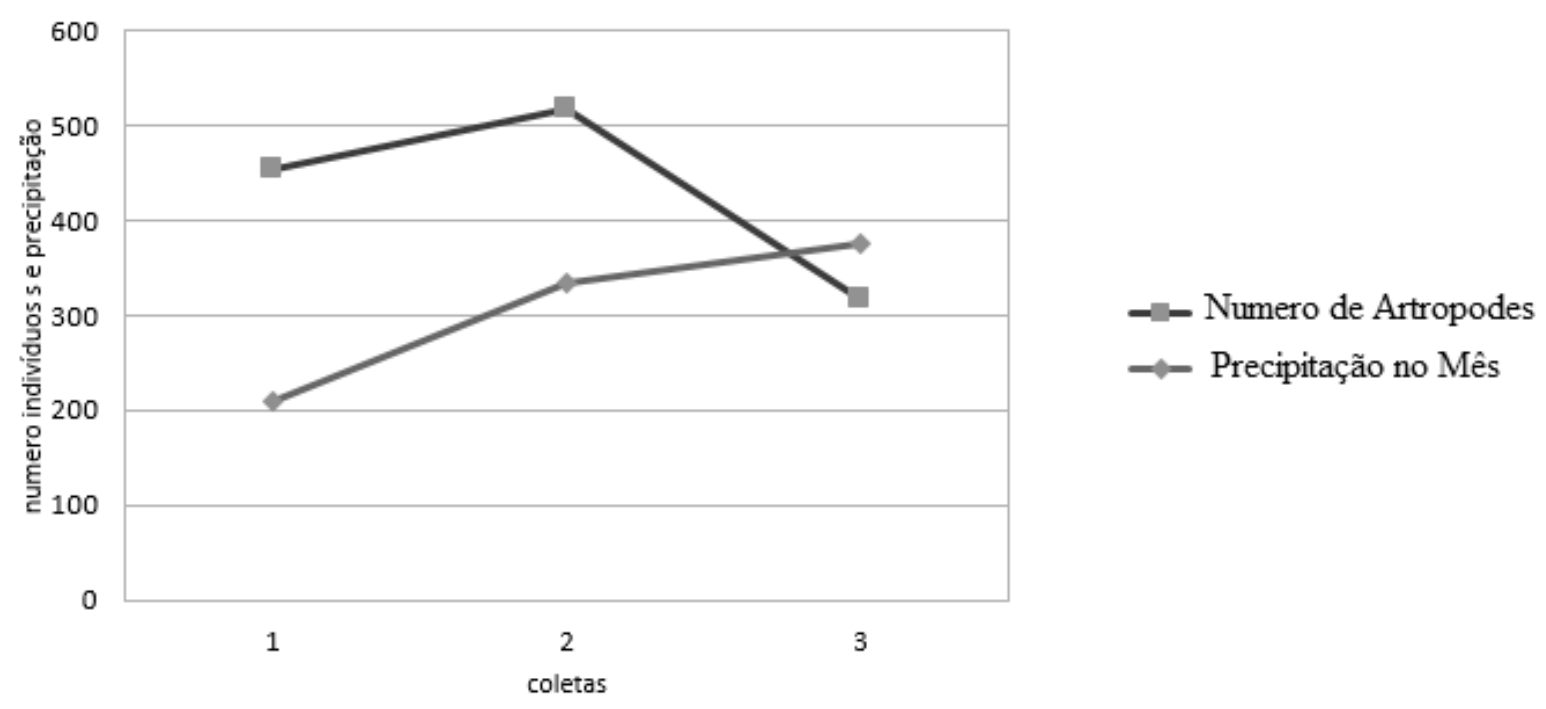

FIGURA 1 - Número total de indivíduos capturados nas armadilhas em cada coleta e precipitação acumulada $(\mathrm{mm})$ do mês.

Ao analisar os resultados, pode-se inferir que o número de insetos possivelmente tenha sido influenciado pelo estádio de desenvolvimento da planta, pois observou-se valores distintos de indivíduos capturados entre as coletas. Quando as plantas de abobrinha estavam em torno dos 18 dias, primeira coleta, estas estavam em pleno crescimento, com a área foliar em desenvolvimento, tendo sido neste momento capturados 455 insetos, o segundo maior valor. $\mathrm{Na}$ segunda coleta, realizada em torno dos 46 dias após o transplantio, as plantas de abobrinha estavam com sua área foliar totalmente desenvolvida e em plena floração, 
apresentando alguns frutos em estágio inicial de desenvolvimento. Esse ambiente propiciou o maior número de insetos capturados durante a pesquisa. Já a terceira coleta foi realizada com 65 dias, nesse período as plantas já estavam com grande carga de frutos e apresentavam ataque de doenças e no final do ciclo. Essa coleta resultou no menor número de insetos capturados, provavelmente devido aos fatores elencados acima.

\section{CONCLUSÕES}

As armadilhas utilizadas, Pitfall e Moericke, foram eficientes na captura de artrópodes das Ordens: Coleoptera, Collembola, Diptera, Hemiptera e Hymenoptera.

A armadilha do tipo Moericke capturou maior diversidade de insetos no início do desenvolvimento da cultura, aos 18 dias. Já, a armadilha do tipo Pitfall apresentou maior diversidade de insetos quando a cultura estava em plena floração, aos 46 dias.

\section{REFERÊNCIAS}

BELLINI, B. C.; ZEPPELINI, D. Registros da fauna de Collembola (Arthropoda, Hexapoda) no Estado da Paraíba, Brasil. Revista Brasileira de Entomologia. v. 53, n.3, p. 386-390, set. 2009

COMÉRIO, E. F.; BENASSI, V.L.R.M.; PERIOTO, N.W. Influência de plantas invasoras na abundância de himenópteros parasitoides (Insecta, Hymenoptera) coletados em cultura de coqueiro anão verde, em Linhares, ES, Brasil. Arquivos do Instituto Biológico. v.80, p.117-123, 2013.

GALLO, D.; NAKANO, O.; SILVEIRA NETO, S.; CARVALHO, R. P. L.; BATISTA, G. C.; BERTI FILHO, E.; PARRA, J. R. P.; ZUCCHI, R. A.; ALVES, S. B.; VENDRAMIN, J. D. Manual de Entomologia Agrícola. 3 ed. São Paulo: Ed. Ceres. 2002. 649 p.

GARCIA, F. R. M. Zoologia Agrícola: Manejo Ecológico de Pragas. Porto Alegre: Editora Rígel. 2008. 256p.

IBGE. Instituto Brasileiro de Geografia e Estatística. Censo Agropecuário 2006. ISSN 0103-6157. Rio de Janeiro: IBGE, 2009. Disponível em:<http://www.ibge.gov.br/home/estatistica/economia/agropecuaria/censoagro/brasi I_2006/Brasil_censoagro2006.pdf> Acesso em 21 nov. 2014.

LARA, R.I.R.; FERNANDES, D.R.R.; VERSUTI, D.R.; TANGO, M.F.A.; PERIOTO, N.W. Sampling and diversity of hymenoptera (Insecta) in an orange orchard/Brazilian Savannah fragment interface. EntomoBrasilis. v.8, p.51-57, 2015.

LARA, R.I.R.; PERIOTO, N.W.; MIRANDA, N.F.; FERNANDES, D.R.R.; MARTINELLI, N.M. Novos registros de Entedoníneos (Hymenoptera, Eulophidae) para a cultura do café (Coffea arabica L.). Coffee Science. v.6, p.242-244, 2011.

LARA, R.I.R.; PERIOTO, N.W.; RAMIRO, Z.A. Número mínimo de armadilhas de AGRARIAN ACADEMY, Centro Científico Conhecer - Goiânia, v.4, n.7; p.183 2017 
Möericke em amostragem de himenópteros parasitóides na cultura da soja Glycine $\max ($ L.) Merrill. Arquivos do Instituto Biológico. v.76, p.55-59, 2009.

MASCARENHAS, M.H.T.; OLIVEIRA, V.R.; SIMÕES, J.C.; RESENDE, L.M.A. Abobrinha Italiana (Cucurbita pepo L. var. melopepo). In: PAULA JÚNIOR, T. J.; VENZON, M. (Org.). 101 Culturas: Manual de tecnologias agrícolas. Belo Horizonte: Epamig, 2007. p. 45-48.

MICHEREFF FILHO, M.; MACHINI, W.D.; MENDONÇA, J.L.; FONSECA, M.E.N.; FERNANDES-ACIOLI, N.A.; BOITEUX, L.S. Resposta à mosca-branca (Bemisia tabaci) e ao Tomato severe rugose virus de acessos de Solanum subgênero Leptostemonum. Horticultura Brasileira. Brasília, v.30, p. 440-445, 2012.

MOREIRA, F. M. S.; HUISING, E. J.; BIGNELL, D. E. O inventário da diversidade biológica do solo: conceitos e orientações gerais. In: SWIFT M. J.; BIGNELL, D. E.; MOREIRA, F. M. S.; HUISING, E. J. Manual de biologia dos solos tropicais. Lavras: UFLA, 2010.

R CORE TEAM. "R": A language and environment for statistical computing. $\mathbf{R}$ Foundation for Statistical Computing, Vienna, Áustria, 2016.

SILVA, L.N.; AMARAL, A.A. Amostragem da mesofauna e macrofauna de solo com armadilha de queda. Revista Verde de Agroecologia e Desenvolvimento Sustentável. v. 8, n. 5, p.108-115, dez. 2013.

TANGO, M.F.A.; FERNANDES. D.R.R.; PAZ, C.C.P.; LARA, R.I.R.; PERIOTO, N.W. Orgilinae (Hymenoptera: Braconidae) em cultivo de café de Cravinhos, SP, Brasil. Revista Colombiana de Entomologia. v.40, p.25-33, 2014.

VILELA, E. F.; DELLA LUCIA, T. M. C. Comunicação química. In: Della Lucia, T. M. C. (ed.). As formigas cortadeiras. Viçosa, Minas Gerais, 1994, p.106-123. 